\title{
The expression of the soluble isoform of hFlt3 ligand by recombinant vaccinia virus enhances immunogenicity of the vector
}

\author{
K. ZURKOVA ${ }^{1}$, K. BABIAROVA ${ }^{1}$, P. HAINZ ${ }^{1}$, J. KRYSTOFOVA ${ }^{1}$, \\ L. KUTINOVA ${ }^{1}$, P. OTAHAL ${ }^{2}$ and S. NEMECKOVA ${ }^{1}$ \\ ${ }^{1}$ Institute of Hematology and Blood Transfusion, Department of Experimental Virology, U Nemocnice 1, CZ-128 20 Prague 2; \\ ${ }^{2}$ Institute of Molecular Genetics, Czech Academy of Sciences, Vídenská 1083, CZ-14220 Prague 4, Czech Republic
}

Received December 9, 2008; Accepted February 19, 2009

DOI: $10.3892 /$ or_00000359

\begin{abstract}
Recombinant vaccinia viruses (rVACV) expressing various tumor-associated antigens have been shown to elicit anti-tumor effect in numerous experimental models and clinical trials. We tested the hypotheses that rVACV expressing biologically active fms-like tyrosine kinase 3 ligand (Flt3L) would show higher immunogenicity than control viruses expressing only model antigen and that coexpression of Flt3L would influence anti-tumor activity of rVACV in the preventive and therapeutic arrangements of the in vivo experiment. To answer these questions, we took advantage of the well-described model of transplanted tumor cells expressing HPV16 E6 and E7 oncoproteins. To determine the effects of hFlt3L on the induction of anti-tumor immunity, we generated live vaccinia viruses that express human Flt3L regulated by the early $\mathrm{H} 5$ or strong synthetic E/L promoter together with fusion protein SigE7LAMP, which is a highly immunogenic form of HPV E7 oncoprotein. We tested Flt3L production in vitro and in vivo. Despite higher expression of Flt3L from the synthetic E/L promoter in vitro, the P13-E/L-FL-SigE7LAMP induced lower levels of Flt3L in the serum of mice than P13-H5-FL-SigE7LAMP. The Flt3L expression under the strong early VACV H5 promoter is able to inhibit expansion of $\mathrm{CD} 11 \mathrm{~b}^{+} \mathrm{Gr}-1^{+}$myeloid suppressor cells
\end{abstract}

Correspondence to: Dr Sarka Nemeckova, Institute of Hematology and Blood Transfusion, U Nemocnice 1, CZ-128 20 Prague 2, Czech Republic

E-mail: sarkan@uhkt.cz

Abbreviations: CTL, cytotoxic T lymphocyte; DC, dendritic cell; Flt3L, Fms like tyrosine kinase 3 ligand; HPV, human papilloma virus; LAMP, lysosome associated membrane protein; NK, natural killer; pS2S, preS2 + surface antigen of hepatitis B virus, PCR, polymerase chain reaction; $\mathrm{VACV}$, vaccinia virus

Key words: vaccinia virus, soluble Flt3 ligand, immune response, CTL, antibodies, HPV16 E7
(MSC) and increase the amount of CD11 b $\mathrm{b}^{+} \mathrm{CD} 11 \mathrm{c}^{+}$dendritic cells in the spleen of mice immunized with vaccinia virus. Determination of viral DNA isolated from the ovaries of infected animals did not reveal differences in replication between rVACVs in this organ. Coexpression of Flt3L by replication-competent virus P13-FL-SigE7LAMP induced enhancement of the cellular immune response against HPV16 E7 and VACV E3 proteins as well as increased anti-tumor efficacy in both the protective and therapeutic immunization schemes. On the other hand, the short-time Flt3L coexpression by MVA-H5-FL-SigE7LAMP was not sufficient to enhance anti-tumor effect of immunization.

\section{Introduction}

Vaccinia virus (VACV) infection induces the release of proinflammatory cytokines and chemokines resulting in stimulation of innate immunity and Th1 biased antigen specific $\mathrm{T}$ cell responses effective in the elimination of virus infected cells or tumor cells. Therefore, recombinant vaccinia virus vectors encoding defined viral or tumor-associated antigens are widely studied as therapeutic anti-viral and anticancer vaccines. It is well established that the immunotherapeutic capacity of the vaccinia vector could be enhanced if biological adjuvants such as cytokines, chemokines or costimulatory molecules were expressed by the same rVACV vector as the antigen. These effects on immunotherapy of tumors were observed with macrophage colony-stimulating factor (GM-CSF) (1), CD40L (2), chemokine MIP3 $\alpha /$ CCL20 (3) or secondary lymphoid organ chemokine CCL21 (4).

One of the possible mechanisms that leads to enhanced immunogenicity of such rVACV is that soluble factors produced by rVACV directly activate professional APCs, which then better stimulate rVACV-induced immune responses. This is a well established mechanism observed following immunization with rVACV expressing GM-CSF which is a key cytokine for DC development in mice. Another cytokine which is similarly important for DC development and maturation in mice, is Fms-like tyrosine kinase 3 ligand (Flt3L) (5). Flt3L is a growth factor that stimulates in synergy with other cytokines (SCF, IL-3, GM-CSF) the proliferation 

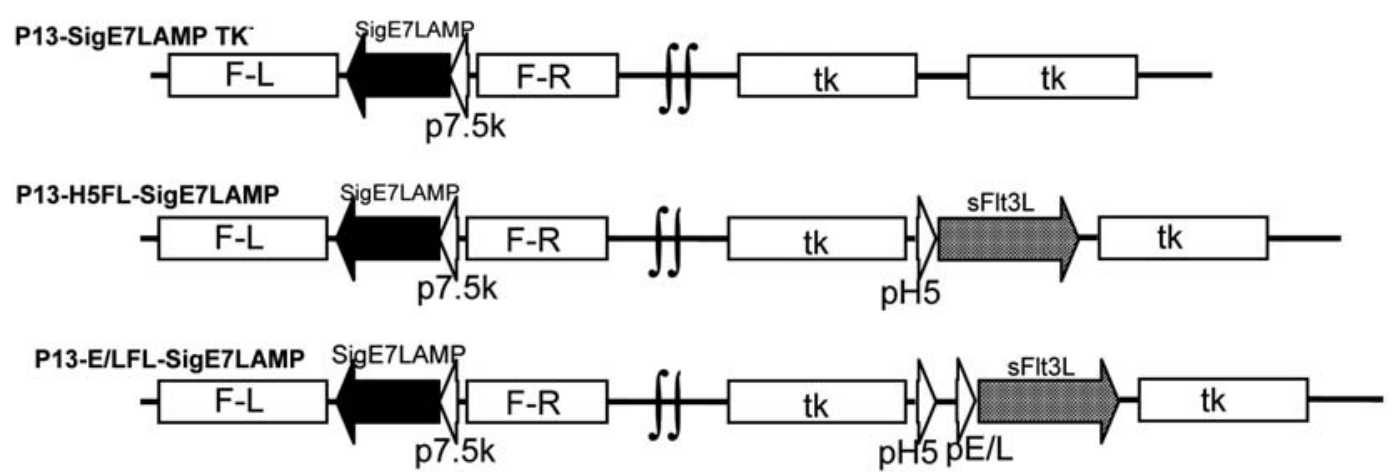

Figure 1. Genome structure of rVACV expressing SigE7LAMP and Flt3L. SigE7LAMP fusion gene was inserted in the non-essential F7L locus behind the $7.5 \mathrm{k}$ early/late promoter (p7.5k). The Flt3L coding sequence was inserted into thymidine kinase locus (TK) and its expression was controlled either by the early/late $\mathrm{H} 5$ promoter (pH5) or a strong synthetic early/late promoter (pEL). In the P13-SigE7LAMP virus the TK gene was inactivated by insertion of a non-coding DNA sequence.

and differentiation of primitive hematopoietic cells. The soluble and biologically active form of Flt3L is generated by cleavage of the primary membrane-associated translation product. The human and mouse Flt3L extracellular domains are very similar and therefore administration of hFlt $3 \mathrm{~L}$ to mice results in the expansion of dendritic cells and increased frequencies of natural killer cells and $\mathrm{B}$ and $\mathrm{T}$ lymphocytes. hFlt3L also enhances DC-mediated immunogenicity in mice (6) and augments antitumor immunity via activation of $\mathrm{CD}^{+}$ T cells or NK cells $(7,8)$.

Persistent infection with high-risk human papilloma viruses (HPVs) can result in intraepithelial neoplasia and malignant lesions of the lower genital tract. HPVs are also suspected to be involved in the induction of other tumors (9) such as head and neck cancer and skin tumors in immunosuppressed persons. Papilloma viruses encode several early proteins such as E2, E5, E6 and E7 which appear as potential target antigens for therapeutic vaccines. So far, immunization with live recombinant vaccinia virus encoding HPV-16,18 E6 and E7 has been shown to induce immune responses and have clinical effects in patients with HPV-associated neoplasia $(10,11)$.

Based on these facts, we decided to test the hypothesis that rVACV expressing biologically active Flt3L would show higher immunogenicity than control viruses expressing only a model antigen. Second, we wanted to assess how coexpression of Flt3L would influence anti-tumor activity of rVACV in the preventive and therapeutic arrangements of the in vivo experiment. To answer these questions, we took advantage of the well-described model of transplanted tumor cells expressing HPV16 E6 and E7 oncoproteins. Immunization with rVACV expressing HPV E7 leads to sufficient immunity to render mice resistant to subsequent challenge with E7-expressing tumor cells under optimal conditions. Thus, to determine the effects of hFLT3 on the induction of anti-tumor immunity, we generated live vaccinia viruses that express human Flt3L together with HPV E7 oncoprotein and determined their immunogenicity in mice. Our results might help in the development of new vaccinia virus-based vaccines and provide new information on Flt3L as an immune adjuvant.

\section{Material and methods}

Recombinant vaccinia viruses ( $r V A C V s$ ). The coding sequence of a soluble isoform of Flt3 ligand (ID number - U29874) was expressed under the control of the early H5 promoter or strong synthetic $\mathrm{E} / \mathrm{L}$ promoter by the recombinant vaccinia virus strain Praha, clone 13 (Fig. 1) (12). The plasmid pHUFLT3L obtained from Immunex (now a wholly owned subsidiary of Amgen) was digested with EcoRI and StuI and the fragment containing the cDNA of Flt3 ligand was ligated with pSC59-H5 (13) cleaved with the same enzymes yielding the pSC59-E/L-FL plasmid. Removal of the E/L promoter from this plasmid by subsequent digestion with BamHI and EcoRI, Klenow fragment treatment, and joining the molecule with T4 DNA ligase resulted in the second plasmid named pSC59-H5-FL. The plasmids were used for transfer of Flt3-L into the VACV genome in the thymidine kinase locus (TK) by the method described previously (14). For evaluation of the effect of Flt3-L on immunogenicity and immunotherapeutic potential of viral vectors against the growth of tumors, we prepared double recombinants where the E7 oncoprotein of human papillomavirus 16 in a highly immunogenic form of SigE7LAMP fusion protein is also coexpressed. In double recombinants, the Flt3-L coding sequence was inserted into vaccinia virus P13-SigE7LAMP expressing the fusion gene SigE7LAMP (Sig-signal sequence of the lysosomeassociated membrane protein 1 (LAMP1), E7-early protein of HPV16, LAMP-transmembrane and intracellular domain of LAMP1) (15). The viruses were denoted as P13-H5-FLSigE7LAMP and P13-E/L-FL-SigE7LAMP. The control virus P13-SigE7LAMP TK- was prepared by recombination with plasmid $\mathrm{p} 11 \mathrm{~K}$ which contains the TK gene disrupted by the $11 \mathrm{k}$ promoter and the multiple cloning site. The virus P13-pS2S with expression of hepatitis B virus envelope antigen was described previously (16). Recombinant P13 viruses were grown in BSC40 cells. Viruses were purified by sucrose-gradient centrifugation (17) and titrated in CV-1 cells.

Detection of Flt3L. Human Flt3L in culture media and mouse sera was detected by ELISA (R\&D Systems Inc.). To detect intracellular Flt3L, Hela cells infected with virus were 
resupended in $1 \mathrm{ml}$ of fresh medium and lysed by freezing and thawing. Released Flt3L was determined in the supernatant separated by centrifugation.

Cells. HPV16 E6E7-expressing TC-1 cells, derived from C57BL/6 mouse (18), were kindly provided by T.C. Wu (Baltimore). BSC40, CV-1 and TC-1 cell lines were cultured in modified E-MEM (EPL) medium containing bovine serum growth-active proteins but no complete serum (19).

Virus infection of mice. Six-week-old C57B1/6 (H-2 $\left.{ }^{\mathrm{b}}\right)$ female mice were obtained from Charles River, Germany. Animals were maintained under standard conditions at the National Institute of Public Health (Prague). The experiments were performed in compliance with Acts Nos. 246/92 and 77/2004 on animal protection against cruelty and Decree No. 311/97 of the Ministry of Health of the Czech Republic, on the care and use of experimental animals. Mice were injected intraperitoneally (i.p.) with $0.5 \mathrm{ml}$ or intramuscularly (i.m.) with $0.1 \mathrm{ml}$ PBS containing sonicated suspension of rVACV.

Quantitative PCR ( $Q-P C R)$. Mice were anesthetized with halothane (Narcotane, Léciva, Praha) and sacrificed. The ovaries were dissected, washed in PBS and homogenized in $0.4 \mathrm{ml}$ PBS using an Omni tissue homogenizer TH-220 (Cole-Parmer) with disposable probe tips, $65 \mathrm{~mm}$. DNA was extracted from $50 \mu 1$ of homogenate by using the tissue protocol of the DNeasy Tissue Kit (Qiagen, Hilden, Germany). To produce RNA-free DNA the samples were treated for 2 min with $4 \mu \mathrm{l}$ of RNase A $(100 \mathrm{mg} / \mathrm{ml})$ added prior to the addition of buffer AL. Real-time quantitative PCR was performed on $2 \mu 1$ of total DNA in a $20-\mu 1$ reaction volume, $0.2 \mathrm{mM}$ dNTPs, $5 \mathrm{mM} \mathrm{MgCl}$, forward primer 5'-TTG GCA ATA GTC CGT GTA ATC-3' (300 nM), reverse primer 5'-ATA CTT AAT CAT GTC GCC GAC-3' (300 nM), and a Taqman probe 5'-CCA GTA ACA CGA GCT GGT GCT TGC TAG-3' BHQ1-FAM (250 nM). The reaction was analyzed by using the real-time quantitative PCR apparatus Rotorgene 3000, Corbett Research (Cambridge, UK). Plasmid DNA containing the amplified fragment was used to construct the standard curve and to quantitate the amount of viral DNA. The amount of viral DNA was normalized to the total amount of DNA in each sample. The detection limit was 7 genome copies per $100 \mathrm{ng}$ of total DNA. Correlation analysis of VACV DNA and Flt3L in mouse samples was calculated by the non-parametric Spearman test.

Assays of immune response. T cell responses were detected as described previously (15). In brief, the spleens were removed from mice 12 days after the immunization. The response of IFN- $\gamma$-producing cells was determined by ELISPOT-IFN $\gamma$ assay upon restimulation with $\mathrm{H}-2 \mathrm{D}^{\mathrm{b}}$ restr icted peptides derived from HPV16 E7 ${ }_{(49-57)}$ (RAHYNIVTF) and VACV E3 $3_{(140-148)}$ proteins for 6 days in vitro. The values obtained for individual mice were analyzed by the unpaired t-test. E7-specific CD8 ${ }^{+}$CTLs were detected by tetramerstaining assay using the R-phycoerythrin labeled $\mathrm{H}-2 \mathrm{D}^{\mathrm{b}}$ / E7 ${ }_{(49-57)}$ tetramer reagent (PeliMer, Sanquin, NL). The stained cells were analyzed on a Coulter-Epics XL flow cytometer (Beckman Instruments). The mean fluorescence values obtained for peptide-restimulated and control cells were analyzed by the unpaired t-test. Detection of VACV specific antibodies was described earlier (20).

Flow cytometry detection of surface molecules on splenocytes. The spleens were mechanically disrupted and a single-cell suspension was acquired after red blood cell lysis using ACK buffer (0.15 $\mathrm{M} \mathrm{NH}_{4} \mathrm{Cl}, 10 \mathrm{mM} \mathrm{KHCO}$ and $0.1 \mathrm{mM}$ EDTA). Cells were stained with fluorescently conjugated mAbs CD3-FITC, CD4-FITC, CD8-FITC, CD11b-PE, CD11bFITC, CD11c-PE, F4/80-PE and Gr-1(Ly6G-FITC). F4/80$\mathrm{PE} \mathrm{mAb}$ was purchased from ABD-Serotec and the other mAbs were supplied by BD PharMingen (San Diego, USA). Expression of surface molecules was determined by flow cytometry using a Coulter-Epics XL flow cytometer (Beckman Instruments) and FlowJo (Tree Star, Inc., Ashland, USA) software.

Tumor induction. To induce the tumors, the TC- 1 cells were injected subcutaneously (s.c.) into the back of C57Bl/6 mice. Tumor growth was measured weekly. Differences between survival curves were statistically analyzed by twoway ANOVA. All statistical analyses were performed using Prism 4.0 software (GraphPad Software, Inc, San Diego, USA).

\section{Results}

Expression of hFlt3-L from recombinant VACV. To enhance immunogenicity of recombinant VACV, we constructed recombinant VACVs that express the Flt3L coding sequence. As the murine and human Flt3L cross-react, we decided to use the human Flt3L soluble isoform. The expression was controlled either by the early VACV H5 promoter or strong synthetic $\mathrm{E} / \mathrm{L}$ promoter. We initially examined hFlt3L production in vitro using human FL-ELISA. Hela cells $\left(1.5 \times 10^{5}\right)$ were infected with P13-H5-FL-SigE7LAMP or P13-E/L-FL-SigE7LAMP at MOI 1 and cultured in $1 \mathrm{ml}$ D-MEM. The production of intracellular and secreted Flt3L was followed up to $30 \mathrm{~h}$ post infection (p.i.) (Fig. 2A). The total amounts of hFlt3L produced were $143 \mathrm{pg} / 10^{3}$ cells and $540 \mathrm{pg} / 10^{3}$ cells for P13-H5-FL-SigE7LAMP and P13-E/LFL-SigE7LAMP viruses, respectively. At a late stage, infection with P13-E/L-FL-SigE7LAMP resulted in a higher proportion of intracellular Flt3L compared to P13-H5-FL-SigE7LAMP with no observed accumulation of Flt $3 \mathrm{~L}$ inside cells.

In the next step, we determined the concentration of hFlt3-L in sera of mice infected intraperitoneally with $1 \times 10^{6}$ PFU (Fig. 2B). The mean concentrations of hFlt3-L on day 4 following virus administration were $92.3 \pm 31.3 \mathrm{pg} / \mathrm{ml}$ and 49.3 $\pm 16.3 \mathrm{pg} / \mathrm{ml}$ for P13-H5-FL-SigE7LAMP and P13-E/LFL-SigE7LAMP, respectively. On the following day, the hFlt3-L levels decreased and on day 6 all sera were negative. In repeated experiments we observed increase of hFlt3L levels in serum following infection with the peak on day 4 . Control viruses without hFlt3L gene elicited no increase of $\mathrm{hFlt3L}$ concentration in serum at any interval after infection.

VACV multiplication in vivo. As we found a great difference in hFlt3-L production by P13-E/L-FL-SigE7LAMP after infection in vitro and in vivo, in the next step, we tested 

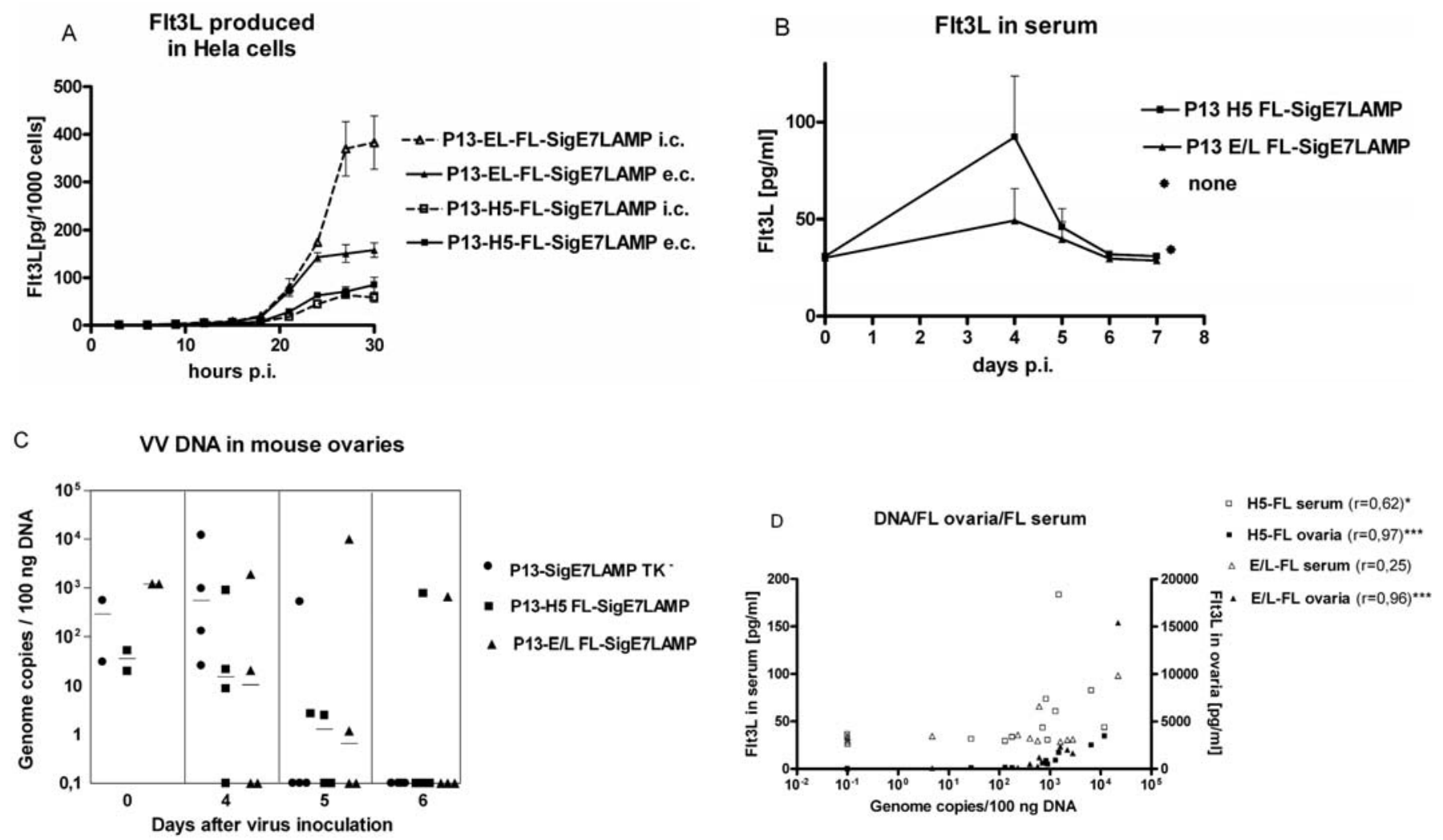

Figure 2. Characterisation of VACV expressing hFlt3L. (A) Production of hFlt3L in vitro was determined in Hela cells infected at MOI 1 with either P13E/L-FL-SigE7LAMP or P13-H5-FL-SigE7LAMP Flt3L. Concentration of hFlt3L was determined by ELISA in cell lysate and in medium at various intervals after infection. (B) Production of hFlt3L in vivo was determined by ELISA in sera of 6-week-old C57B1/6 (H-2 $\left.{ }^{b}\right)$ female mice inoculated i.p. with $1 \times 10^{6} \mathrm{PFU}$ of either P13-E/L-FL-SigE7LAMP or P13-H5-FL-SigE7LAMP on days 0, 4, 5, 6 and 7 p.i. (C) Replication of rVACV in the ovaries was determined in mice inoculated with P13-SigE7LAMP TK-, P13-H5-FL-SigE7LAMP or P13-E/L-FL-SigE7LAMP as in (B). DNA was determined in the ovaries by Q-PCR on days $0,4,5$ and 6. (D) The animals from experiment (B) were also examined for VACV DNA and Flt3L in ovarian extracts. The graph shows correlation analysis between Flt3L in the serum and ovaries and multiplication of VACV in the ovaries in samples from days 4, 5 and 6.

whether hFlt3-L expression affected multiplication of recombinant viruses in vivo. The replication of viruses was quantified by Q-PCR assay in DNA isolated from the mouse ovaries. Mice were injected i.p. with $1 \times 10^{6} \mathrm{PFU}$ and the ovaries were collected on subsequent days. The results of repeated experiments were similar. The amount of viral DNA in ovaries increased gradually from inoculation to day 4 and then dropped. Comparison of multiplication of different viruses on days $0,4,5$ and 6 is shown in Fig. 2C. The co-expression of the hFlt3L gene did not affect significantly multiplication of rVACV which expressed the SigE7LAMP fusion construct as the second insert. Distinct amounts of DNA detected repeatedly on day 0 were due to an increased amount of defective virus particles present in the inoculum. Multiplication of both Flt3L-expressing viruses correlated well (Fig. 2D) with the FLt3L produced in the ovaries on days 4, 5 and $6(\mathrm{r}=0.96-0.97 ; \mathrm{p}<0.0001)$. Multiplication of P13-H5FL-SigE7LAMP also positively correlated with Flt3L found in mouse serum $(\mathrm{r}=0.62 ; \mathrm{p}=0.03)$ whereas release of Flt3L expressed by P13-E/L-FL-SigE7LAMP seemed to be suppressed because concentration of Flt3L in serum did not correlate with the amount of virus DNA in ovaries $(r=0.25$, $\mathrm{p}=0.4)$.

Coexpression of $h F l t 3-L$ increased the immune response induced by rVACVs in mice. We assessed whether hFlt3L had any effect on specific $\mathrm{CD}^{+} \mathrm{T}$ cell responses against antigens expressed by the same virus. Mice were injected i.p. with a single dose of $3 \times 10^{6}$ PFU of P13-SigE7LAMP TKP13-H5-FL-SigE7LAMP or P13-E/L-FL-SigE7LAMP. The splenocytes were collected at two intervals: 12 days or 5 weeks after infection. The cells were cultured for 6 days with immunodominant nona-peptides derived from papilloma virus 16 early protein $\mathrm{E} 7_{(49-57)}$ and from vaccinia virus early protein $\mathrm{E} 3_{(140-148)}$. The $\mathrm{T}$ cell response was determined by ELISPOT-IFN $\gamma$ and E7-tetramer assays. As shown in Fig. 3, coexpression of hFlt3L increased the frequency of antigenspecific T cells detected by ELISPOT-IFN $\gamma$ (A) and tetramer (B) assays when collected 12 days after immunization. However, the enhancing effect of hFlt3-L coexpression on cellular immunity was only observed at the early interval. Five weeks after immunization, similar HPV16-E7-specific response to restimulation with peptide was induced by all three recombinants $(\mathrm{C}$ and $\mathrm{D})$. To find out whether hFlt3L coexpression also affected the humoral immune response against vaccinia virus proteins, the mouse sera were tested by ELISA for the presence of anti-VACV antibodies (Fig. 3E). The mean absorbance values were $0.30 \pm 0.09 \mathrm{SE}, 0.27 \pm 0.04$ SE and 0.32 \pm 0.06 SE for P13-SigE7LAMP TK-, P13-H5-FLSigE7LAMP and P13-E/L-FL-SigE7LAMP, respectively. Though no significant differences in total anti-VACV immunoglobulin were observed, antibody responses elicited by P13- 
A

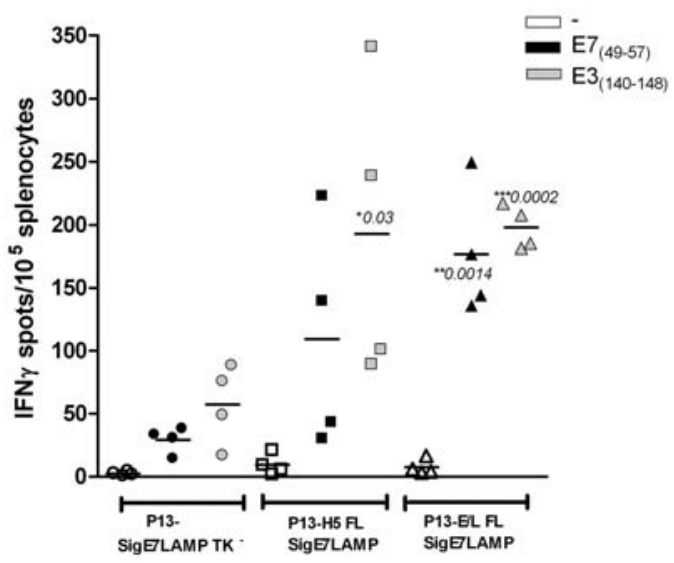

C

\section{ELISPOT IFN $\gamma$}

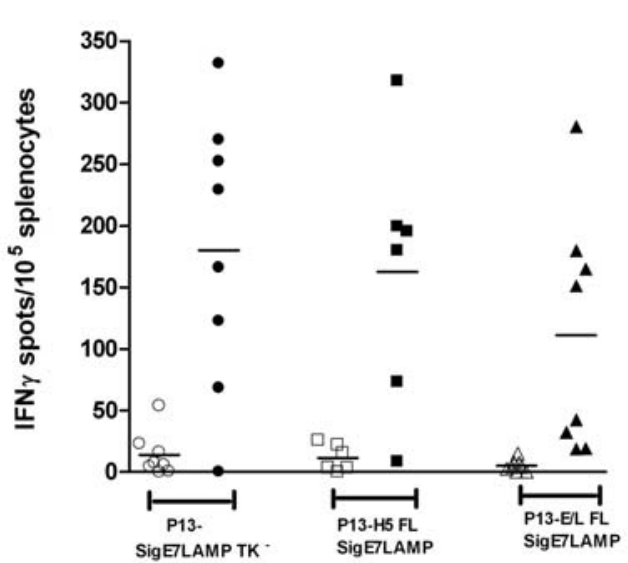

$B$

Tetramer

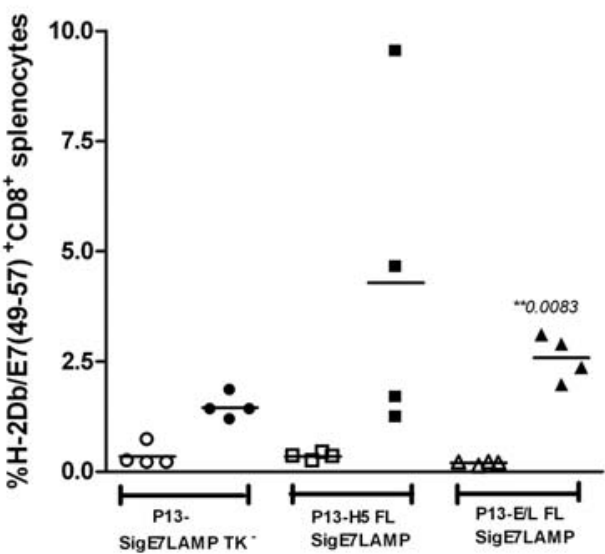

D

Tetramer

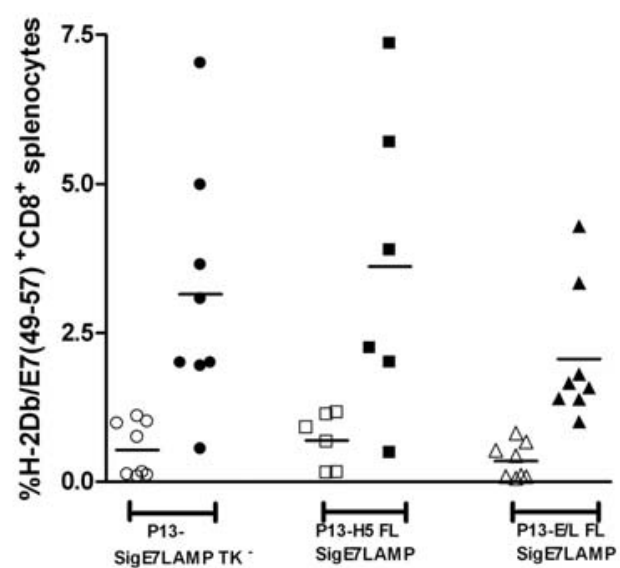

E

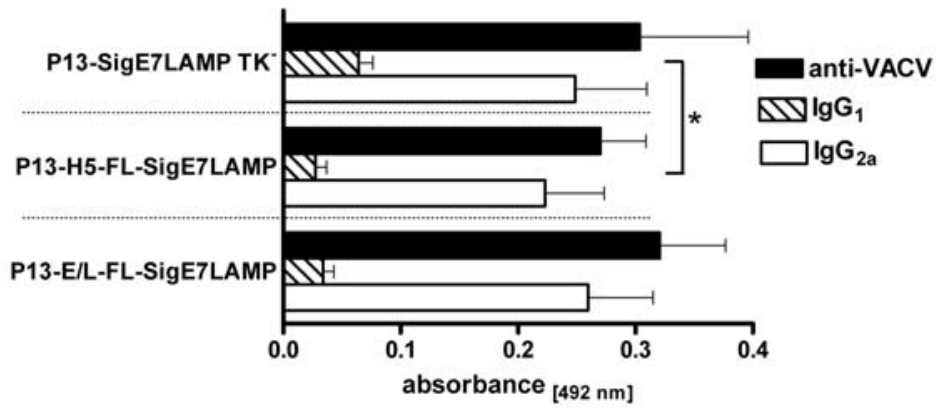

Figure 3. T cell response induced in mice by immunization with rVACVs expressing hFlt3L. Six-week-old C57B1/6 (H-2 ${ }^{b}$ ) female mice were injected i.p. with one dose $3 \times 10^{6}$ PFU of P13-SigE7LAMP TK- P13-H5-FL-SigE7LAMP or P13-E/L-FL-SigE7LAMP. The response of CD8 ${ }^{+}$T cell lymphocytes specific for HPV16-E7 (full symbols) and VACV-E3 protein (shaded symbols) in individual mice was determined either 12 days (A and B) or 5 weeks (C and D) later by IFN- $\gamma$ ELISPOT (A and C) and tetramer (B and D) assays. The antibody response against VACV was measured in sera collected 5 weeks after virus administration $(\mathrm{E})$. The columns represent mean \pm SEM. The data were analyzed by two-way ANOVA. P-values stand for significance of the difference from the P13-SigE7LAMP TK- virus.

H5-FL-SigE7LAMP virus were the lowest. When we analysed $\mathrm{IgG}$ isotypes in the anti-VACV humoral immune response, we found again that P13-H5-FL-SigE7LAMP elicited a significantly lower IgG1 response than P13-SigE7LAMP TKcontrol virus.
Coexpression of hFlt3L improved preventive and therapeutic immunization against the growth of TC-1 tumors. Given the significant enhancement that we found in the T cell response, we examined whether hFlt3L coexpression during immunization could also affect protection against the subsequent 
A
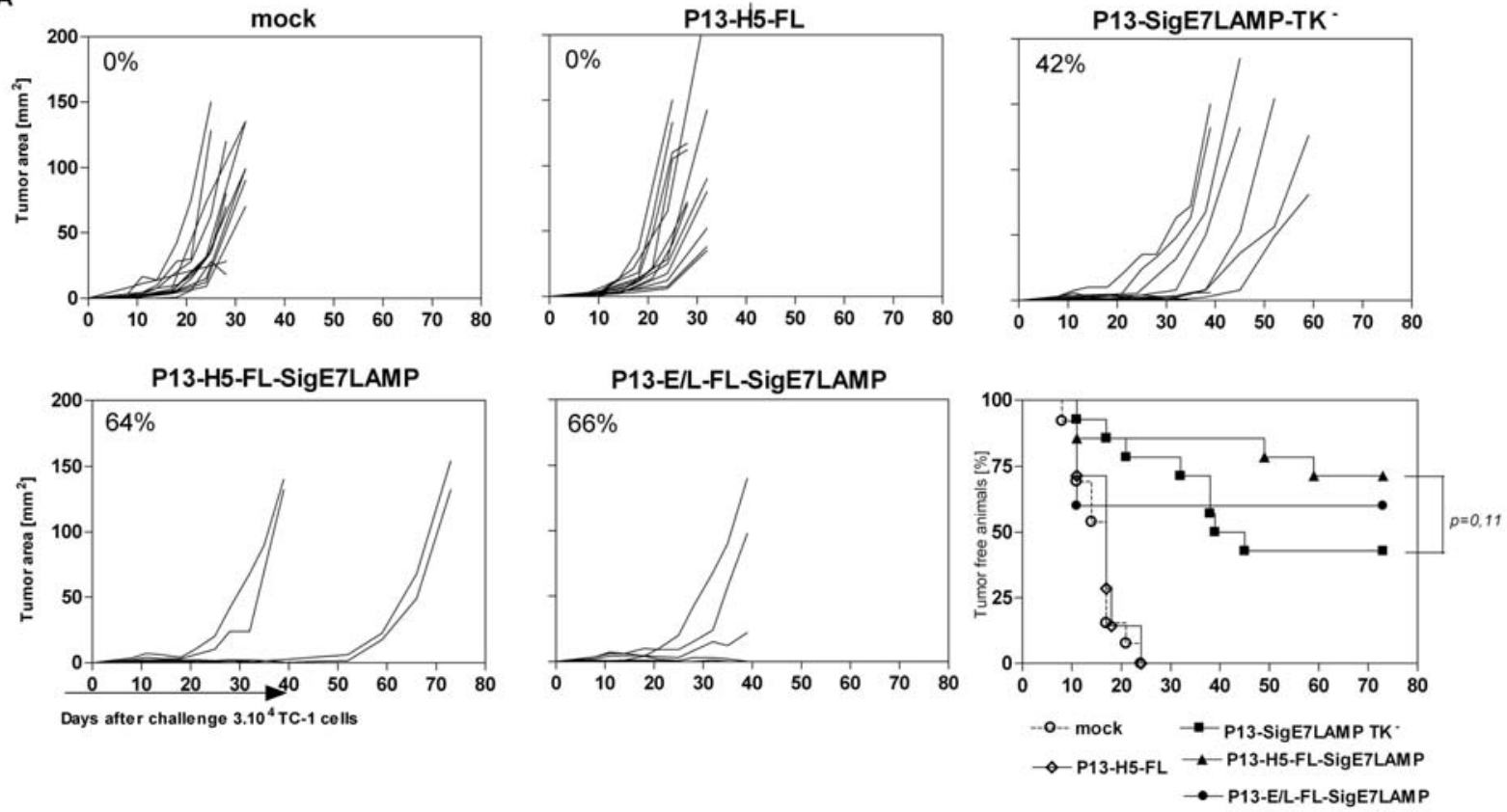

B
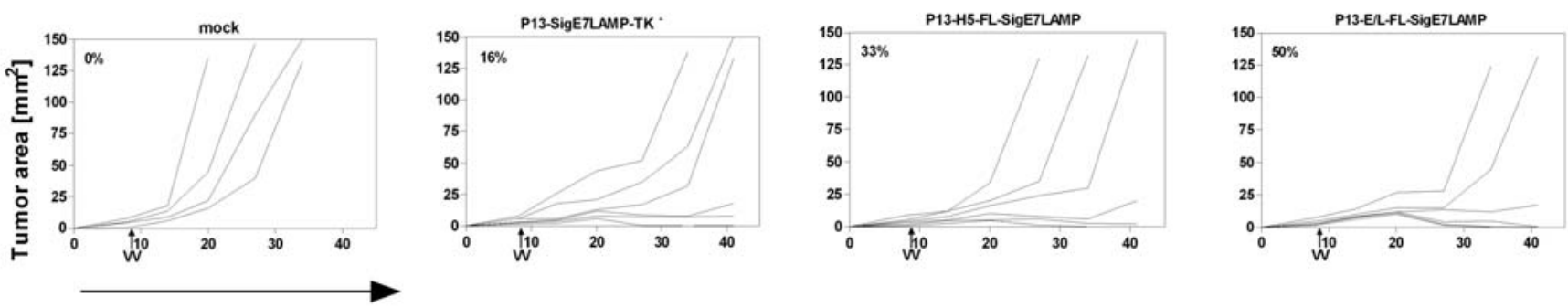

Days after challenge $6.10^{4} \mathrm{TC}-1$ cells

Figure 4. The effect of hFlt3L coexpression on the protective and therapeutic anti-tumor effects of immunization with rVACV. (A) Six-week-old C57Bl/6 $\left(\mathrm{H}-2^{\mathrm{b}}\right)$ female mice $(\mathrm{n}=14)$ were injected i.m. with one dose of 1x106 PFU of either P13-SigE7LAMP TK- P13-H5-FL-SigE7LAMP, P13-E/L-FLSigE7LAMP, P13-H5-FL or mock infected. Two weeks after immunization, the animals were challenged s.c. with $3 \times 10^{4}$ TC-1 tumor cells and the growth of tumors was monitored up to 73 days. (B) For evaluation of the therapeutic efficacy of double recombinants, mice (n=6) were injected s.c. with $6 x 10^{4} \mathrm{TC}-1$ cells. Nine days later, the animals were once immunized i.p. with 1.106 PFU P13-SigE7LAMP TK-, P13-H5-FL-SigE7LAMP or P13-E/L-FL-SigE7LAMP or mock infected. The growth of tumors in individual animals is shown in separate graphs for each group and the fraction of tumor-free mice at the end of the experiment is indicated in the upper left corner of each graph. The data were statistically analyzed by comparison of survival curves of tumor-free animals (A) using the log-rank test. Representative results from two out of four independent challenge experiments are shown.

formation of TC-1 tumors expressing the E7 oncoprotein and whether treatment with the double recombinants could induce regression of already formed tumors. In the preventive immunization scheme, mice were immunized with a single i.m. dose of $1 \times 10^{6} \mathrm{PFU} \mathrm{rVACV}$ and they were challenged with $3 \times 10^{4}$ TC- 1 cells s.c. two weeks later. Immunization with P13-H5-FL-SigE7LAMP significantly inhibited tumor formation and slowed tumor growth compared with the P13SigE7LAMP TK ${ }^{-}$virus (survival curve, $\mathrm{p}=0.11$ ) (Fig. 4A). Inoculation of P13-H5-FL virus, which does not express E7 antigen, had no effect on the growth of tumors in comparison with mock-infected mice. Next, we determined the effect of hFlt3L coexpression on therapeutic immunization with rVACV. Mice were injected s.c. with $6 \times 10^{4}$ TC- 1 cells 9 days later. When every mouse had a tumor, a single i.p. dose of $1 \times 10^{6} \mathrm{PFU} \mathrm{rVACV}$ was given. Immunization with all three recombinants inhibited tumor growth; nevertheless, the highest tumor regression (3/6) was induced by P13-E/LFL-SigE7LAMP eventhough the differences were not statistically significant. Also P13-H5-FL-SigE7LAMP increased the proportion of tumor-free mice (2/6) in comparison with the P13-SigE7LAMP TK- virus (1/6) (Fig. 4B).

Coexpression of hFlt3L under $H 5$ promoter protected mice from expansion of myeloid $\mathrm{CD} 11 \mathrm{~b}^{+} \mathrm{Gr}-1^{+}$cells. To determine the effect of hFlt3L expressed by P13-SigE7LAMP on the immune cells, we quantified the major subpopulations of cells and assessed their maturation status in the spleens of infected mice by flow cytometry using surface markers CD3, CD4, CD8, CD11b, CD11c, F4/80 and Gr-1(Ly6G) on days $4,8,12,20$ and 35 after administration of $1 \times 10^{6}$ PFU rVACV. Injection of each of the viruses induced an increase in spleen cellularity on days $4(193 \%)$ and $8(174 \%)$ comparatively to non-injected mice $(100 \%)$. The spleens 

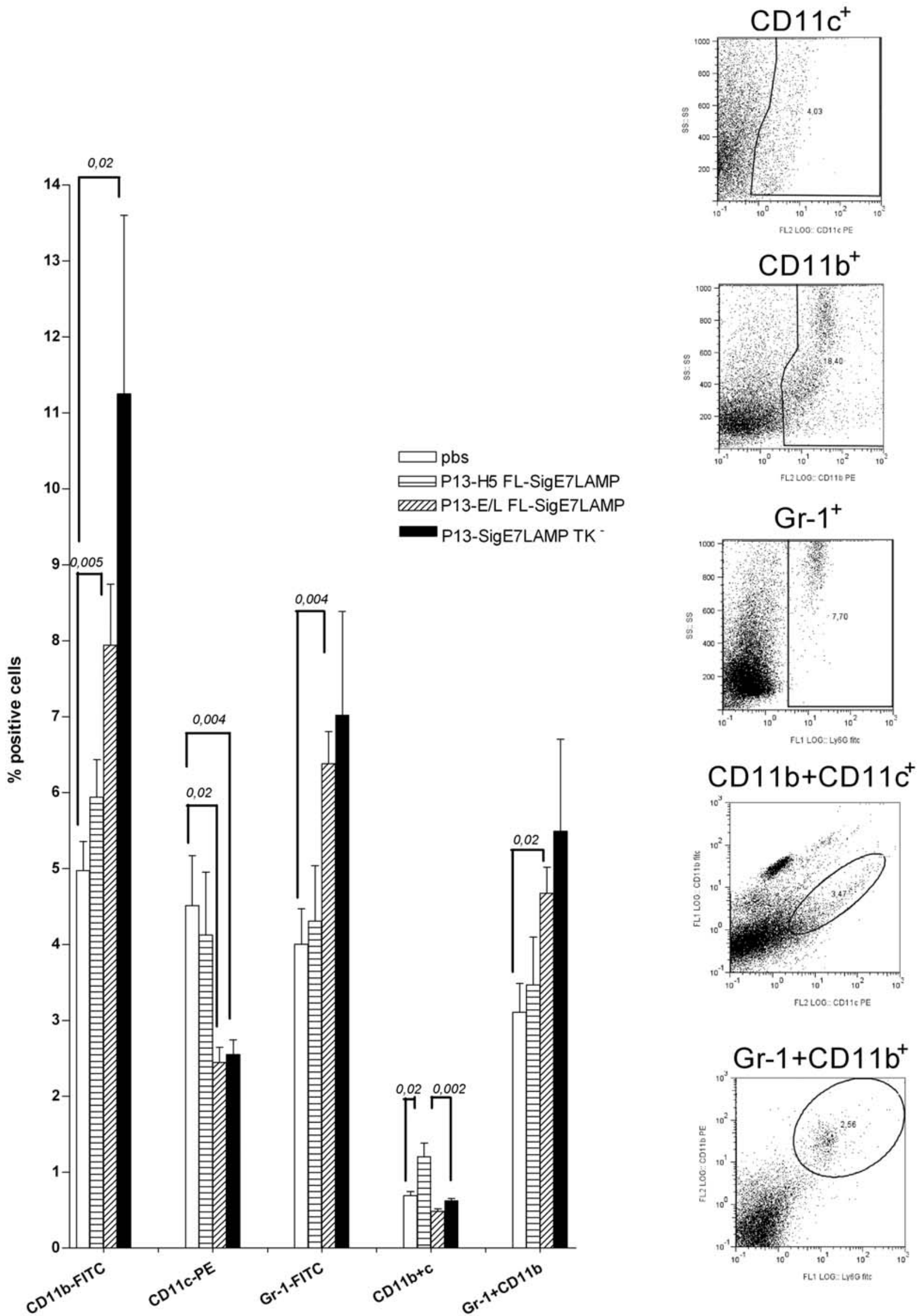

Figure 5. The effect of Flt3L coexpression on surface molecules of splenocytes of infected mice. Six-week-old C57Bl/6 (H-2b) female mice (n=6) were inoculated i.p. with 1x106 PFU of P13-H5-FL-SigE7LAMP, P13-E/L-FL-SigE7LAMP, P13-SigE7LAMP TK- or mock infected. Splenocytes were collected and analyzed by flow cytometry 5 weeks after infection using Gr-1(Ly6G), CD11b and CD11c specific MAbs. The p-values represent significant differences. Insets on the right side show examples of detection of specific markers on the surface of cells. 
normalized by day 12 . All three viruses induced temporary depletion of $\mathrm{CD}^{+}$cells during the phase of intensive virus multiplication between days 4 and 8 , which was followed by an increase in $\mathrm{CD}^{+}$counts on day 20 that exceeded non-significantly those in naïve mice found on day 0 . The population of $\mathrm{Gr}-1^{+}, \mathrm{CD} 11 \mathrm{~b}^{+}$and double positive cells in the 3 groups did not significantly change in comparison with naïve mice until day 20 . However, an increase in $\mathrm{Gr}-1^{+}$, $\mathrm{CD}_{1} 1 \mathrm{~b}^{+}$and double positive cells was observed 5 weeks after infection with P13-E/L-FL-SigE7LAMP which induced low levels of Flt3L in serum only and with P13-SigE7LAMP TK- control virus. On the other hand, the production of Flt3L by P13-H5-FL-SigE7LAMP protected animals from expansion of Gr-1+ and CD11 ${ }^{+}$cells (Fig. 5). Moreover, P13-H5-FL-SigE7LAMP virus also induced an increase in the $\mathrm{CD} 11 \mathrm{~b}^{+} \mathrm{CD} 11 \mathrm{c}^{+}$cell population which is referred to as spleen dendritic cells. The other surface molecules CD3, $\mathrm{CD} 8$, and F4/80 were not influenced by virus infection at any interval.

\section{Discussion}

In this study, we examined the immunomodulating effect of Flt3L expression and its impact on therapeutic anti-cancer activity of immunization with vaccinia virus recombinants expressing E7 antigen of human papilloma virus 16.

Recombinant vaccinia viruses expressing GM-CSF, a growth factor of myeloid dendritic cells, have already been tested with success in clinical immunotherapy of tumors. For the same purpose, we have constructed vaccinia virus recombinants expressing Flt3L which is known to induce expansion of plasmacytoid dendritic cells in vitro and to have adjuvant and anti-tumor activity in vivo. The gene encoding the secretory form of Flt3L was regulated by two promoters of different strengths during the early and late periods of infection. We observed that despite very high expression of Flt3L from the synthetic E/L promoter in vitro, the P13E/L-FL-SigE7LAMP induced low levels of Flt3L in serum of inoculated mice. The peak of virus DNA in the ovaries coincided with the maximum serum Flt3L levels and appeared 4-5 days after infection of mice. It has been shown earlier that high levels of IL12 produced by recombinant vaccinia virus suppressed substantially virus multiplication, which resulted in lower cytokine production in vivo than in vitro $(21,22)$. To decide whether low Flt3L production by P13E/L-FL-SigE7LAMP could be explained by this mechanism, we quantitated viral DNA isolated from the ovaries of infected animals and found the replication levels of all three viruses to be similar. We have not observed significantly different levels of VACV-specific antibodies in mice infected either with P13-E/L-FL-SigE7LAMP or P13-SigE7LAMP which can be regarded as a second indirect evidence of unrestricted multiplication in vivo. We have demonstrated that coexpression of the hFlt3L coding sequence has not significant effect on multiplication of recombinant vaccinia virus expressing SigE7LAMP.

Next, we characterized the adjuvant activity of Flt3L when coexpressed by a replication-competent vaccination vector. We found that the response of antigen specific CD8 ${ }^{+}$ $\mathrm{T}$ cells expressed by the double recombinant was enhanced by coexpression of Flt3L and that the enhancing effect depended on the amount of Flt3L released from infected cells in vivo. P13-H5-FL-SigE7LAMP, which produced the highest amount of Flt3L in vivo, was the most immunogenic of all three viruses. An enhanced $\mathrm{CD} 8^{+}$response was found to the peptide derived from SigE7LAMP protein which was expressed under the $7.5 \mathrm{k}$ early/late promoter, and also to VACV E3 protein that is controlled by the early promoter of the E3L gene. It was noted, that enhancing effect of Flt3L was higher in population of functional IFN $\gamma$ secreting cells, detected by ELISPOT, than in population positive for phenotypic markers in tetramer assay, which could include exhausted and anergic CD8 ${ }^{+} \mathrm{T}$ cells. The enhanced response of $\mathrm{CD}^{+} \mathrm{T}$ cells was accompanied by a lower antibody response particularly of $\mathrm{IgG} 1$ isotype. Such findings indicate that Flt3L coexpression shifted the immune response elicited by VACV towards Th1 type. In the present study, the enhancing effect of Flt3L was observed with replicating virus where the cytokine expression spanned several days. We also prepared double recombinant MVA-H5-FL-SigE7LAMP (not shown) that only insignificantly enhanced the cellular response. Similar results for Flt3L-expressing MVA were recently reported by Chavan et al (3). We have shown previously that in vivo expression of another cytokine (IL12) directed by non-replicating MVA-IL12 virus usually ceases several hours after infection, whereas cytokine expression driven from replicating P13-IL12 can be sustained for several days (21). It is known that the major expansion of DC1 and stimulation of Th1 subset require repeated administration of Flt3L, preferably for 10 days, since a single dose of Flt3L has no effect (23). Analogously, our results indicate that enhancing effects of Flt3L coexpression cannot be expected after administration of a single dose of non-replicating poxvirus but only when replicating virus is used for expression, with Flt3L concentration still increased five days after infection as reported here.

The enhancement that we found in the $\mathrm{CD}^{+} \mathrm{T}$ cell response against E7 oncoprotein of HPV16, correlated with the anti-tumor effect against the growth of TC-1 tumors in mice. From previous experiments, we knew that immunization with $10^{6}$ PFU of P13-SigE7LAMP virus i.p. completely inhibited the growth of TC-1 tumors if administered either two weeks before the challenge or one day after immunization. Therefore, we utilized the less effective intramuscular route for preventive immunization, more suitable for use in larger animals and humans. In the therapeutic experiments, the treatment by i.p. injection started nine days after the challenge when all animals had already developed tumors with a mean area of $4.19 \mathrm{~mm}^{2}$ (SEM=0.4). Treatment with Flt3Lexpressing recombinants induced regression of tumors smaller than $4.0 \mathrm{~mm}^{2}$ whereas larger tumors of $4-10 \mathrm{~mm}^{2}$ escaped the therapy.

Intraperitoneal injection of any of the three recombinants caused a temporary increase in spleen cellularity comparatively to non-injected mice. Differences in expression of several lineage markers on splenocytes were not significant except for Gr-1, CD11b and CD11c molecules. At a late interval, we observed increased $\mathrm{CD} 11 \mathrm{~b}^{+} \mathrm{Gr}-1^{+}$populations in the spleen of animals infected with control P13-SigE7LAMP virus. The $\mathrm{CD} 11 \mathrm{~b}^{+} \mathrm{Gr}-1^{+}$population was less abundant after P13-E/L- 
FL-SigE7LAMP and was the smallest after immunization with P13-H5-FL-SigE7LAMP which was the best Flt3L producer. Recombinant vaccinia virus is known to induce immunosuppression of adaptive responses mediated by a specialized population of myeloid suppressor cells (MSC) that express Gr-1 and CD11b markers (24). The decrease or absence of MSCs in the secondary lymphoid organ could contribute to the enhancement of immunogenicity and anti-tumor effects of rVACV expresing the Flt3L genes. The second significant difference was the increase in $\mathrm{CD} 11 \mathrm{~b}^{+} \mathrm{CD} 11 \mathrm{c}^{+}$dendritic cells, induced only by immunization with P13-H5-FL-SigE7LAMP virus.

In summary, our results suggest that immunogenicity and anti-tumor activity of VACV expressing tumor antigen HPV16 E7 could be enhanced by coexpression of the soluble form of Flt3L. The Flt3L expression under the strong early VACV H5 promoter is able to inhibit expansion of MCS in the immunized mice and to increase the amount of dendritic cells, which could contribute to anti-tumor effect of immunization.

\section{Acknowledgements}

This work was supported by grants NR 9238-3/2007 and MZOUHKT2005 of the Ministry of Health of the Czech Republic and grant 310/05/H5333 of the Grant Agency of the Czech Republic.

\section{References}

1. Kim JH, Oh JY, Park BH, Lee DE, Kim JS, Park HE, Roh MS Je JE, Yoon JH, Thorne SH, Kirn D and Hwang TH: Systemic armed oncolytic and immunologic therapy for cancer with JX-594, a targeted poxvirus expressing GM-CSF. Mol Ther 14: 361-370, 2006 .

2. Feder-Mengus C, Schultz-Thater E, Oertli D, Marti WR, Heberer M, Spagnoli GC and Zajac P: Non-replicating recombinant vaccinia virus expressing CD40 ligand enhances APC capacity to stimulate specific CD4+ and CD8+ $\mathrm{T}$ cell responses. Hum Gene Ther 16: 348-360, 2005.

3. Chavan R, Marfatia KA, An IC, Garber DA and Feinberg MB: Expression of CCL20 and granulocyte-macrophage colonystimulating factor, but not Flt3-L, from modified vaccinia virus ankara enhances antiviral cellular and humoral immune responses. J Virol 80: 7676-7687, 2006.

4. Flanagan K, Glover RT, Horig H, Yang W and Kaufman HL: Local delivery of recombinant vaccinia virus expressing secondary lymphoid chemokine (SLC) results in a CD4 $\mathrm{T}$ cell dependent antitumor response. Vaccine 22: 2894-2903, 2004.

5. Suss G and Shortman K: A subclass of dendritic cells kills CD4 T cells via Fas/Fas-ligand-induced apoptosis. J Exp Med 183: 1789-1796, 1996.

6. Pulendran B, Smith JL, Jenkins M, Schoenborn M, Maraskovsky E and Maliszewski CR: Prevention of peripheral tolerance by a dendritic cell growth factor: flt3 ligand as an adjuvant. J Exp Med 188: 2075-2082, 1998.

7. Lynch DH, Andreasen A, Maraskovsky E, Whitmore J, Miller RE and Schuh JC: Flt3 ligand induces tumor regression and antitumor immune responses in vivo. Nat Med 3: 625-631, 1997.

8. Fernandez NC, Lozier A, Flament C, Ricciardi-Castagnoli P, Bellet D, Suter M, Perricaudet M, Tursz T, Maraskovsky E and Zitvogel L: Dendritic cells directly trigger NK cell functions: cross-talk relevant in innate anti-tumor immune responses in vivo. Nat Med 5: 405-411, 1999.
9. zurHausen H: Papillomaviruses in human cancers. Proc Assoc Am Physicians 111: 581-587, 1999

10. Davidson EJ, Boswell CM, Sehr P, Pawlita M, Tomlinson AE McVey RJ, Dobson J, Roberts JS, Hickling J, Kitchener HC and Stern PL: Immunological and clinical responses in women with vulval intraepithelial neoplasia vaccinated with a vaccinia virus encoding human papillomavirus 16/18 oncoproteins. Cancer Res 63: 6032-6041, 2003.

11. Baldwin PJ, van der Burg SH, Boswell CM, Offringa R, Hickling JK, Dobson J, Roberts JS, Latimer JA, Moseley RP, Coleman N, Stanley MA and Sterling JC: Vaccinia-expressed human papillomavirus 16 and 18 e 6 and e 7 as a therapeutic vaccination for vulval and vaginal intraepithelial neoplasia. Clin Cancer Res 9: 5205-5213, 2003.

12. Kutinova L, Ludvikova V, Simonova V, Otavova M Krystofova J, Hainz P, Press M, Kunke D and Vonka V: Search for optimal parent for recombinant vaccinia virus vaccines. Study of three vaccinia virus vaccinal strains and several virus lines derived from them. Vaccine 13: 487-493, 1995.

13. Nemeckova S, Stranska R, Subrtova J, Kutinova L, Otahal P, Hainz P, Maresova L, Sroller V, Hamsikova E and Vonka V: Immune response to E7 protein of human papillomavirus type 16 anchored on the cell surface. Cancer Immunol Immunother 51: 111-119, 2002

14. Perkus ME, Panicali D, Mercer S and Paoletti E: Insertion and deletion mutants of vaccinia virus. Virology 152: 285-297, 1986.

15. Mackova J, Kutinova L, Hainz P, Krystofova J, Sroller V, Otahal P, Gabriel P and Nemeckova S: Adjuvant effect of dendritic cells transduced with recombinant vaccinia virus expressing HPV16-E7 is inhibited by co-expression of IL12. Int J Oncol 24: 1581-1588, 2004

16. Kutinova L, Nemeckova S, Hamsikova E, Zavadova H, Ludvikova V, Broucek J, Kunke D, Konig J, Zakharova LG and Pashvykina GV: Hepatitis B virus proteins expressed by recombinant vaccinia viruses: influence of preS2 sequence on expression surface and nucleocapsid proteins in human diploid cells. Arch Virol 134: 1-15, 1994.

17. Joklik WK: The purification of four strains of poxvirus. Virology 18: 9-18, 1962.

18. Lin KY, Guarnieri FG, Staveley-O'Carroll KF, Levitsky HI, August JT, Pardoll DM and Wu TC: Treatment of established tumors with a novel vaccine that enhances major histocompatibility class II presentation of tumor antigen. Cancer Res 56: 21-26, 1996.

19. Michl J: Metabolism of cells in tissue culture in vitro. I. The influence of serum protein fractions on the growth of normal and neoplastic cells. Exp Cell Res 23: 324-330, 1961.

20. Hamsikova E, Zavadova H, Kutinova L, Ludvikova V, Krchnak V, Nemeckova $S$ and Vonka V: Priming effect of recombinant vaccinia virus coding for the middle hepatitis B surface antigen. Arch Virol 113: 283-289, 1990.

21. Nemeckova S, Sroller V, Hainz P, Krystofova J, Smahel M and Kutinova L: Experimental therapy of HPV16 induced tumors with IL12 expressed by recombinant vaccinia virus in mice. Int J Mol Med 12: 789-796, 2003.

22. Gherardi MM, Ramirez JC, Rodriguez D, Rodriguez JR, Sano G, Zavala F and Esteban M: IL-12 delivery from recombinant vaccinia virus attenuates the vector and enhances the cellular immune response against HIV-1 Env in a dose-dependent manner. J Immunol 162: 6724-6733, 1999.

23. Parajuli P, Mosley RL, Pisarev V, Chavez J, Ulrich A, Varney M, Singh RK and Talmadge JE: Flt3 ligand and granulocytemacrophage colony-stimulating factor preferentially expand and stimulate different dendritic and T-cell subsets. Exp Hematol 29: 1185-1193, 2001.

24. Bronte V, Wang M, Overwijk WW, Surman DR, Pericle F, Rosenberg SA and Restifo NP: Apoptotic death of CD8+ T lymphocytes after immunization: induction of a suppressive population of Mac-1+/Gr-1+ cells. J Immunol 161: 5313-5320, 1998. 